\title{
FIBERINGS OF SPHERES AND $H$-SPACES WHICH ARE RATIONAL HOMOLOGY SPHERES
}

\author{
BY WILLIAM BROWDER \\ Communicated by Alex Rosenberg, February 2, 1962
}

There are various fiber bundles known whose total spaces are spheres. Examples are the Hopf maps $S^{3} \rightarrow S^{2}, S^{7} \rightarrow S^{4}, S^{15} \rightarrow S^{8}$ and and fiberings over projective spaces. It is an open question whether these are all the fiberings of spheres with connected fibers.

Spanier and Whitehead [6] showed that in such a fibering the fiber must be an $H$-space. The results of Borel $[2 ; 3]$ showed that the fiber is a rational homology sphere.

THeOREM 1. Let $p: S^{n} \rightarrow B$ be a fiber bundle map onto a polyhedron $B$, with fiber $F$ a connected polyhedron. Then $F$ is the homotopy type of $S^{1}, S^{3}$ or $S^{7}$.

The proof depends on studying the torsion of $H^{*}(F)$, in particular the 2-torsion.

Theorem 2. Let $X$ be an $H$-space, connected, with $H_{i}(X)$ finitely generated for all $i$, zero for large $i$. Suppose in addition that $X$ is a rational homology sphere, i.e., $H_{*}(X ; Q) \cong H_{*}\left(S^{n} ; Q\right)$ for some $n$. If $H^{1}\left(X ; Z_{2}\right)=0$, then

$$
H^{*}\left(X ; Z_{2}\right)=\Lambda(x) \otimes Z_{2}\left[S q^{1} x\right] /\left(S q^{1} x\right)^{2^{q}}, \quad 0 \leqq q<\infty,
$$

$\operatorname{dim} x$ is odd, $>1$.

$$
\text { If } H^{1}\left(X ; Z_{2}\right) \neq 0 \text {, then } H^{*}\left(X ; Z_{2}\right)=Z_{2}[x] / x^{2^{q}}, 1 \leqq q<\infty, \operatorname{dim} x=1 \text {. }
$$

Since $F$ satisfies the hypothesis of Theorem 2, we may apply it to this situation. A spectral sequence argument using Theorem 2, similar to Borel's argument [3, p. 165], yields the result that $H^{*}\left(F ; Z_{2}\right)$ $=\Lambda(x)$, i.e., $F$ is a mod 2 homology sphere. Namely, Theorem 2 shows that $H^{*}\left(F ; Z_{2}\right)$ has a simple system of transgressive generators, and employing a theorem of Borel [3, Proposition 16.1], we get that $H^{*}\left(B ; Z_{2}\right)$ is a polynomial ring on the "transgressions" of the generators, in dimensions $<n$. But now, analyzing the structure of the spectral sequence, we cannot arrive at $E_{\infty} \cong H^{*}\left(S^{n} ; Z_{2}\right)$, unless $H^{*}\left(F ; Z_{2}\right)$ has only one generator; i.e., $H^{*}\left(F ; Z_{2}\right)=\Lambda(x)$.

Adams [1] has shown that a mod 2 homology sphere which is an $H$-space is a 1,3 , or 7 dimensional mod 2 homology sphere, hence a rational homology 1,3 , or 7 sphere. It is then easy to show that an $H$-space which is a rational homology 1,3 , or 7 sphere has no odd tor- 
sion. Hence $F$ is an integral homology sphere, and it follows that $F$ is the homotopy type of a 1,3 , or 7 sphere.

Theorem 2 may also be applied to studying the cohomology of the "projective plane" of an $H$-space $X$, if $X$ is a rational homology sphere. By studying the Steenrod squares in this space one can show that if $S q^{1} x \neq 0$ then $\operatorname{dim} x=1$, from which one can deduce the following:

Theorem 3. Let $X$ be an $H$-space, connected, with $H_{i}(X)$ finitely generated for all $i$, zero for large $i$, and suppose $X$ is a rational homology sphere, i.e., $H_{*}(X ; Q) \cong H_{*}\left(S^{n} ; Q\right)$, for some $n$. Then $X$ is the singular homotopy type of one of the following: $S^{1}, S^{3}, S^{7}, P^{3}$, or $P^{7},\left(P^{i}=\right.$ real projective space of dimension i).

The proofs of these theorems will be found in [4]. In addition, the proof of Theorem 2 relies heavily on some general results on differential Hopf algebras from [5].

\section{REFERENCES}

1. J. F. Adams, On the non-existence of elements of Hopf invariant one, Ann. of Math. 72 (1960), 20-104.

2. A. Borel, Impossibilité de fibrer une sphère par un produit de sphères, C. R. Acad. Sci. Paris 231 (1950), 943-945.

3. - Sur la cohomologie des espaces fibrés principaux et des espaces homogènes de groupes de Lie compacts, Ann. of Math. 57 (1953), 115-207.

4. W. Browder, Higher torsion in H-spaces, (to appear).

5. - On differential Hopf algebras, (to appear).

6. E. H. Spanier and J. H. C. Whitehead, On fibre spaces in which the fibre is contractible, Comment. Math. Helv. 29 (1955), 1-8.

Cornell University 